TRANSACTIONS OF THE

AMERICAN MATHEMATICAL SOCIETY

Volume 351, Number 9, Pages 3823-3842

S 0002-9947(99)02146-7

Article electronically published on April 27, 1999

\title{
THE TRACE SPACE AND KAUFFMAN'S KNOT INVARIANTS
}

\author{
KEQIN LIU
}

\begin{abstract}
The traces in the construction of Kauffman's knot invariants are studied. The trace space is determined for a semisimple finite-dimensional quantum Hopf algebra and the best lower bound of the dimension of the trace space is given for a unimodular finite-dimensional quantum Hopf algebra.
\end{abstract}

\section{INTRODUCTION}

It is well known that quantum groups have been successfully used in the construction of Witten-Reshetikhin-Turaev invariants of 3-manifolds ([4] and [10]). Recently, there have been some remarkable new developments in this direction. By employing right integrals on certain Hopf algebras, Kauffman and Radford have constructed a new class of invariants of 3-manifolds ([2] and [3]), which are called Kauffman-Radford-Hennings invariants of 3-manifolds.

At the core of Kauffman-Radford-Hennings invariants of 3-manifolds are Kauffman's knot invariants. In fact, the Kauffman-Radford-Hennings invariant of a 3 -manifold is just a scalar multiple of Kauffman's knot invariant. It is also now known that the construction of Kauffman's knot invariant depends only on the algebraic structure of a class of associative algebras called quantum algebras and is independent of the representation theory of quantum algebras. Because of these new properties it is easier to appreciate the amazing connection between the deep algebraic structures of Hopf algebras and the topological invariants of knots, links and 3-manifolds.

A key ingredient in the construction of Kauffman's knot invariants is the trace on a quantum algebra. The trace can be produced from a right integral for $H^{*}$, where $H$ is a unimodular finite-dimensional ribbon Hopf algebra ([2], [3] and [7]). The traces produced from right integrals for a finite-dimensional Hopf algebra are linearly dependent since such right integrals are unique up to a scalar. The construction of Kauffman's knot invariants clearly indicates that for two of Kauffman's knot invariants produced from two traces to be differen, the two traces must be linearly independent. Therefore, an important question in the study of Kauffman's knot invariants is this: how many linearly independent traces are there on a quantum algebra? The purpose of this paper is to discuss this question. The set of all traces on a quantum algebra form a vector space, which is called the trace space for the quantum algebra. In this paper, we will determine the trace space for a semisimple finite-dimensional quantum Hopf algebra and give a best lower bound

Received by the editors March 25, 1996 and, in revised form, April 21, 1997.

1991 Mathematics Subject Classification. Primary 17B35, 17B37, 17C50, 18A10, 57M25, $57 \mathrm{~N} 10$.

(C)1999 American Mathematical Society 
for the dimension of the trace space for a unimodular finite-dimensional quantum Hopf algebra.

This paper is organized as follows. In section 1 we present Radford's algorithm for computing Kauffman's knot invariant using the combinatorial description of a knot diagram given in [7]. From the algorithm we can see how the algebraic structure of a quantum algebra with a trace is used in the construction of Kauffman's knot invariants. In section 2 we recall some basic definitions and important properties about integrals on a finite-dimensional Hopf algebra.

Let $\mathbb{T}_{0}\left(H^{*}\right)$ be the trace space for a quantum algebra $H$. In section 3 we study the trace spaces for a class of quantum algebras called quantum Hopf algebras. An interesting phenomenon is that the trace space $\mathbb{T}_{0}\left(H^{*}\right)$ is a Jordan algebra if $H$ is a quantum Hopf algebra. We determine $\operatorname{dim} \mathbb{T}_{0}\left(H^{*}\right)$ for a semisimple finitedimensional quantum Hopf algebra and give a lower bound of $\operatorname{dim} \mathbb{T}_{0}\left(H^{*}\right)$ for a unimodular finite-dimensional quantum Hopf algebra.

In section 4 we discuss the class of finite-dimensional Hopf algebras $s \ell_{q, z}^{t}(2)$ introduced in [6]. The quantum algebra structures of $s \ell_{q, z}^{t}(2)$ depend on some parametric points $\left(d_{\alpha \beta}\right)_{0 \leq \alpha, \beta<t}$. Every parametric point $\left(d_{\alpha \beta}\right)_{0 \leq \alpha, \beta<t}$ is a point in $\overline{V_{\ell}} \cup \overline{V_{r}}$, where both $\overline{V_{\ell}}$ and $\overline{V_{r}}$ are varieties in $\mathbb{C}^{t^{2}}$. Now $\left(s \ell_{q, z}^{t}(2),\left(d_{\alpha \beta}\right)_{0 \leq \alpha, \beta<t}\right)$ is quasi-triangular if and only if $\left(d_{\alpha \beta}\right)_{0 \leq \alpha, \beta<t} \in \overline{V_{\ell}} \cap \overline{V_{r}}$. Hence the quantum Hopf algebra $\left(s \ell_{q, z}^{t}(2),\left(d_{\alpha \beta}\right)_{0 \leq \alpha, \beta<t}\right)$ is not quasi-triangular for every point $\left(d_{\alpha \beta}\right)_{0 \leq \alpha, \beta<t} \in$ $\left(\overline{V_{\ell}} \cup \overline{V_{r}}\right) \backslash\left(\overline{V_{\ell}} \cap \overline{V_{r}}\right)$. Since $\left(\overline{V_{\ell}} \cup \overline{V_{r}}\right) \backslash\left(\overline{V_{\ell}} \cap \overline{V_{r}}\right)$ is far from empty, the examples of quantum Hopf algebras are much richer than those of quasi-triangular Hopf algebras. By studying integrals, we see that the class of quantum Hopf algebras $s \ell_{q, z}^{t}(2)$ is always unimodular.

In section 5 we determine the trace space for the quantum Hopf algebra $s \ell_{q, z}^{t}(2)$ with odd $t$. It turns out that if $t$ is odd, then the dimension of the trace space for $s \ell_{q, z}^{t}(2)$ is exactly the lower bound given in section 3 . This proves that the lower bound given in section 3 is the best for unimodular finite-dimensional quantum Hopf algebras.

\section{RADFORD'S ALGORITHM FOR COMPUTING KAUFFMAN'S KNOT INVARIANTS}

Kauffman's knot invariants are a class of invariants of regular isotopy of unoriented knots. Using the algebraic description of regular isotopy, Kauffman found that the construction of knot invariants does not depend on the full definition of Hopf algebras and what is needed is just the algebraic structure of a class of associative algebras, which are called quantum algebras by Kauffman in [2].

Definition 1.1. An associative algebra $H$ over a commutative ring $k$ is called a quantum algebra if there exist $S \in \operatorname{Hom}_{k}(H, H), \mathcal{R} \in H \otimes H$ and $G \in H$ such that

1. $S(x y)=S(y x)$ for $x, y \in H$,

2. $\mathcal{R}_{12} \mathcal{R}_{13} \mathcal{R}_{23}=\mathcal{R}_{23} \mathcal{R}_{13} \mathcal{R}_{12}$,

3. $\mathcal{R}^{-1}=(S \otimes 1)(\mathcal{R})=\left(1 \otimes S^{-1}\right)(\mathcal{R})$,

4. $S(G)=G^{-1}$ and $S^{2}(x)=G x G^{-1}$ for $x \in H$,

where $\mathcal{R}_{12}:=\mathcal{R} \otimes 1 \in H \otimes H \otimes H, \mathcal{R}_{13}:=(1 \otimes \sigma)\left(\mathcal{R}_{12}\right), \mathcal{R}_{23}:=(\sigma \otimes 1)\left(\mathcal{R}_{12}\right)$ and $\sigma$ is the twist automorphism of $H \otimes H$ given by $\sigma: x \otimes y \mapsto y \otimes x$ for $x, y \in H$. The quantum algebra $H$ depending on $S, \mathcal{R}$ and $G$ is denoted by $(H, S, \mathcal{R}, G)$. 
The following equations are a useful corollary of (3) in Definition 1.1.

$$
\left(S^{m} \otimes S^{m}\right)\left(\mathcal{R}^{ \pm 1}\right)=\mathcal{R}^{ \pm 1} \quad \text { for } m \in \mathbb{Z}
$$

Definition 1.2. Let $(H, S, \mathcal{R}, G)$ be a quantum algebra. A functional $\operatorname{tr} \in H^{*}:=$ $H_{o m}(H, k)$ is said to be a trace on $H$ if it satisfies the following properties:

1. $\operatorname{tr}(x y)=\operatorname{tr}(y x)$ for $x, y \in H$,

2. $\operatorname{tr}(x)=\operatorname{tr}(S(x))$ for $x \in H$.

Kauffman proved in [2] that a knot invariant can be constructed from a quantum algebra $H$ and a trace on $H$. Radford introduced an algorithm for computing Kauffman's knot invariants in [7]. We need a combinatorial description of a knot diagram to present Radford's algorithm.

A vertical line segment is defined as a closed non-horizontal straight line segment with two different endpoints. An arc is defined as a semicircular arc with endpoints which lie on a horizontal line. The concavity of a semicircular arc has only two possibilities. Hence, if $x$ is an arc, then $x$ is one of the following:

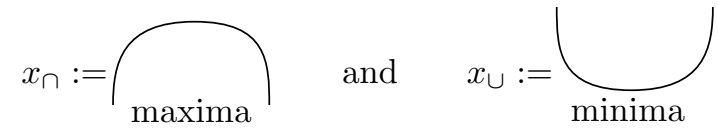

If $x$ and $y$ are two vertical line segments with finite slopes which have opposite signs and $x \cap y=\{p\}$, where $p$ is not an endpoint of either $x$ or $y$, then the ordered pair $(x, y)$ is defined as a crossing when $x$ crosses over $y$. According to whether or not $y$ has positive or negative slope, we have two types of crossing:

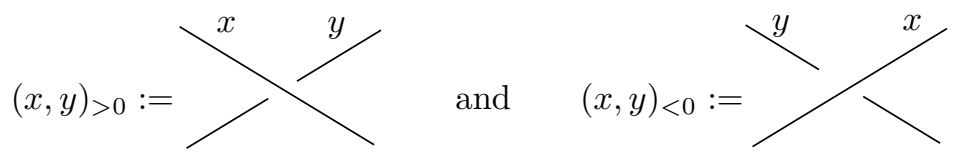

Let $\mathcal{L}$ be a set of vertical line segments. A subset $\mathcal{C} \subseteq \mathcal{L} \times \mathcal{L}$ is called a crossing set if $(x, y) \in \mathcal{C}$ implies $(y, x) \notin \mathcal{C}$, and whenever $(x, y),(w, z) \in \mathcal{C}$ and $(x, y) \neq$ $(w, z),(x, y) \cap(w, z)=\emptyset$.

It is clear that a knot diagram can be expressed as a triple $K=(\mathcal{L}, \mathcal{A}, \mathcal{C})$, where $\mathcal{L}$ and $\mathcal{A}$ are finite sets of vertical line segments and arcs, respectively, and $\mathcal{C} \subseteq \mathcal{L} \times \mathcal{L}$ is a crossing set such that

a) If $x, y \in \mathcal{L} \cup \mathcal{A}$ are different, then $x^{\prime} \cup y^{\prime}$ has two connected components, unless $(x, y) \in \mathcal{C}$ or $(y, x) \in \mathcal{C}$, where $x^{\prime}:=x \backslash\{$ endpoints of $x\}$;

b) $g(\mathcal{L} \cup \mathcal{A})$ is a cyclic graph, where $g(\mathcal{L} \cup \mathcal{A})$ denote the graph whose vertices are the endpoints of the members of $\mathcal{L} \cup \mathcal{A}$ and whose edge between the vertex $p$ and the vertex $q$ is the member of $\mathcal{L} \cup \mathcal{A}$ which has $p$ and $q$ as endpoints;

c) If $x, y \in \mathcal{L} \cup \mathcal{A}$ are different, then the highest and lowest points of $x \cup y$ are not common endpoints;

d) There exists a vertical line segment $x \in \mathcal{L}$ such that $x$ is not a component of a crossing in $\mathcal{C}$.

Conversely, a knot diagram can be reconstructed from a triple as above. Hence, we identify a knot diagram with such a triple.

Let $(H, S, \mathcal{R}, G)$ be a quantum algebra over a commutative ring $k$ and $t r: H \rightarrow k$ be a trace on $H$. For any knot diagram $K$, a scalar $T R(K)$ in $k$ can be constructed from $(H, S, \mathcal{R}, G)$ and $t r$. The scalar $T R(K)$, depending on the quantum algebra structure of $H$ and the trace $\operatorname{tr}$ on $H$, is called a Kauffman's knot invariant for 
$K$. Radford gave a rigorous algorithm to describe the construction of Kauffman's knot invariants in [7].

Let $k\left[t, t^{-1}\right]$ be the Laurent polynomial ring over $k$. Then the associative $k-$ algebra $\mathcal{H}:=k\left[t, t^{-1}\right] \otimes H$ is a free left $k\left[t, t^{-1}\right]$-module. We write $t^{m} h:=t^{m} \otimes h$ for $m \in \mathbb{Z}$ and $h \in H$ and regard both $k\left[t, t^{-1}\right]$ and $H$ as $k$-subalgebras of $\mathcal{H}$. Radford's algorithm for computing $T R(K)$ consists of five steps.

Step 1. Represent a knot diagram $K$ as a triple $K=(\mathcal{L}, \mathcal{A}, \mathcal{C})$, which satisfies the conditions $a), b), c$ ) and $d$ ).

Step 2. Express the cyclic graph $g(\mathcal{L} \cup \mathcal{A})$ in the form

$$
p_{1} \stackrel{x_{1}}{\longrightarrow} p_{2} \stackrel{x_{2}}{\longrightarrow} \ldots \stackrel{x_{n-1}}{\longrightarrow} p_{n} \stackrel{x_{n}}{\longrightarrow} p_{1},
$$

where $\mathcal{L} \cup \mathcal{A}=\left\{x_{1}, \ldots, x_{n}\right\}, p \stackrel{x}{\longrightarrow} q$ denotes the unique edge of $g(\mathcal{L} \cup \mathcal{A})$ joining the vertex $p$ and the vertex $q$, the edge $x_{1}$ satisfies the condition $d$ ) above and the vertex $p_{2}$ is higher than the vertex $p_{1}$.

Step 3. Construct $h_{i}(1 \leq i \leq n)$ in the $n$-fold tensor product $\mathcal{H}^{(n)}:=$ $\mathcal{H} \otimes \cdots \otimes \mathcal{H}$ according to the following rules:

$i)$ If $x_{i} \in \mathcal{L}$ and $\left(x_{i}, x_{j}\right) \notin \mathcal{C}$ for any $j$, set $h_{i}:=\overbrace{1 \otimes \cdots \otimes 1}^{n \text { times }}$, where 1 is the identity of $\mathcal{H}$.

ii) If $x_{i} \in \mathcal{L}$ and $\left(x_{i}, x_{j}\right) \in \mathcal{C}$ for some $j$, set

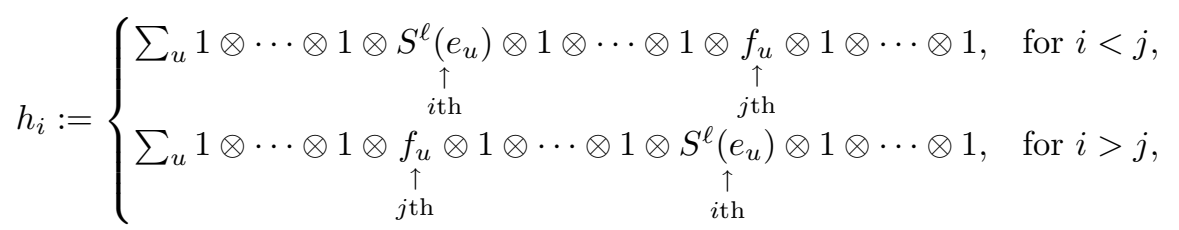

where $\mathcal{R}=\sum_{u} e_{u} \otimes f_{u} \in H \otimes H$ and

$$
\ell:= \begin{cases}0, & \text { if the slope of } x_{j} \text { is positive } \\ 1, & \text { if the slope of } x_{j} \text { is negative. }\end{cases}
$$

iii) If $x_{i} \in \mathcal{A}$, set $h_{i}:=1 \otimes \cdots \otimes 1 \otimes \underset{\substack{\uparrow \\ i \text { th }}}{t^{\ell}} \otimes 1 \otimes \cdots \otimes 1$, where $\ell=1$ or -1

according to whether the direction of the movement through the $\operatorname{arc} p_{i} \stackrel{x_{i}}{\longrightarrow} p_{j}$ from $p_{i}$ to $p_{j}$ is counterclockwise or clockwise.

Step 4. Apply the "straightening" rule:

$$
\cdots \otimes a \otimes t^{\ell} \otimes \cdots \longrightarrow \cdots \otimes t^{\ell} \otimes S^{\ell}(a) \otimes \cdots, \quad \text { where } a \in H
$$

to $h(K):=h_{1} h_{2} \ldots h_{n} \in \mathcal{H}^{(n)}$ to obtain an element $\overline{h(K)} \in k\left[t, t^{-1}\right]^{(|\mathcal{A}|)} \otimes H^{(|\mathcal{A}|)}$.

Step 5. Use the multiplication map $\mathcal{H}^{(n)} \rightarrow \mathcal{H}$ to produce an element $t^{-2 d} \otimes$ $w \in \mathcal{H}$ from the "straightened" tensor sum $\overline{h(K)}$, where $w \in H$. Replacing $t^{-2}$ by $G$ in $t^{-2 d} \otimes w$ gives an element $G^{d} \otimes w \in H \otimes H$. Applying the multiplication in $H$ to $G^{d} \otimes w$, we construct an element $G^{d} w$ of $H$. Kauffman's knot invariant for the $\operatorname{knot} K$ is $T R(K):=\operatorname{tr}\left(G^{d} w\right)$.

Remark 1. It is clear that the element $h(K) \in \mathcal{H}^{(n)}$ produced in Step 4 satisfies $h(K)=h_{i_{1}} h_{i_{2}} \ldots h_{i_{u}}$, where $2 \leq i_{1}<i_{2}<\cdots<i_{u} \leq n$ and either $x_{i_{j}} \in \mathcal{A}$ or $\left(x_{i_{j}}, y\right) \in \mathcal{C}$ for some $y \in \mathcal{L}$. So $u=|\mathcal{A}|+|\mathcal{C}|$. This means that the edges which make a contribution in the construction of $T R(K)$ are arcs and those vertical line segments which are the first components of crossings. 


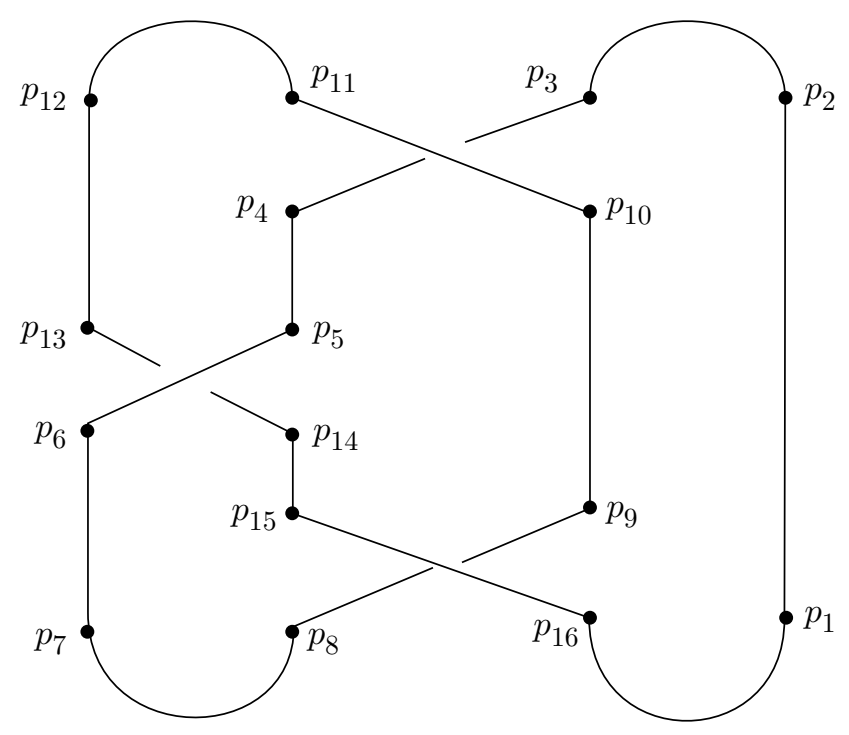

FiguRE 1

Remark 2. The expressions of the cyclic graph $g(\mathcal{L} \cup \mathcal{A})$ in Step 2 are not unique because the edges satisfying the condition $d$ ) are not unique. However, the construction of Kauffman's knot invariants is independent of the choice of the expressions of the cyclic graph $g(\mathcal{L} \cup \mathcal{A})$.

Remark 3. The integer $d$ in $t^{-2 d} \otimes w$ appearing in Step 5 is the Whitney degree of the knot diagram $K$, which is the total turn of the tangent vector to the curve as one traverses it in the direction from $p_{1}$ to $p_{2}$.

As an example, let us construct Kauffman's knot invariant $T R(K)$ for the trefoil knot $K$. The trefoil knot can be represented as a triple $K=(\mathcal{L}, \mathcal{A}, \mathcal{C})$, where

$$
\begin{aligned}
\mathcal{L} \cup \mathcal{A} & =\left\{x_{1}, \ldots, x_{16}\right\}, \\
\mathcal{A} & =\left\{\left(x_{2}\right)_{\cap}^{+},\left(x_{7}\right)_{\cup}^{+},\left(x_{11}\right)_{\cap}^{+},\left(x_{16}\right)_{\cup}^{+}\right\}, \\
\mathcal{C} & =\left\{\left(x_{10}, x_{3}\right)_{>0},\left(x_{5}, x_{13}\right)_{<0},\left(x_{15}, x_{8}\right)_{>0}\right\}
\end{aligned}
$$

and the cyclic graph $g(\mathcal{L} \cup \mathcal{A})$ is expressed as:

$$
p_{1} \stackrel{x_{1}}{\longrightarrow} p_{2} \stackrel{x_{2}}{\longrightarrow} \ldots \stackrel{x_{15}}{\longrightarrow} p_{16} \stackrel{x_{16}}{\longrightarrow} p_{1} .
$$

The notation $x_{\cup}^{+}$(respectively, $x_{\cap}^{+}$) for an $\operatorname{arc} x$ means that the $\operatorname{arc} p \stackrel{x}{\longrightarrow} q$ is a minima (respectively, a maxima) and the direction from $p$ to $q$ is counterclockwise. The diagram for the trefoil knot can be described from the triple above as shown in Figure 1.

It is easy to check that the element $h(K)$ in the 16 -fold tensor product $\mathcal{H}^{(16)}$ is given by

$$
\begin{aligned}
h(K) & =h_{1} h_{2} \ldots h_{16}=h_{2} h_{5} h_{7} h_{! 0} h_{11} h_{15} h_{16} \\
& =\sum_{i, j, k} 1 \otimes t \otimes f_{j} \otimes 1 \otimes S\left(e_{i}\right) \otimes 1 \otimes t \otimes f_{k} \otimes 1 \otimes e_{j} \otimes t \otimes 1 \otimes f_{i} \otimes 1 \otimes e_{k} \otimes t .
\end{aligned}
$$


After applying the "straightening" rule and the multiplication map $\mathcal{H}^{(16)} \rightarrow \mathcal{H}$, we get an element $t^{4} \otimes \sum_{i, j, k} S^{3}\left(f_{j}\right) S^{4}\left(e_{i}\right) S^{2}\left(f_{k}\right) S^{2}\left(e_{j}\right) S\left(f_{i}\right) S\left(e_{k}\right)$. Hence, Kauffman's knot invariant for the trefoil knot $K$ is

$$
T R(K)=\operatorname{tr}\left(G^{-2} \sum_{i, j, k} S^{3}\left(f_{j}\right) S^{4}\left(e_{i}\right) S^{2}\left(f_{k}\right) S^{2}\left(e_{j}\right) S\left(f_{i}\right) S\left(e_{k}\right)\right) .
$$

By (1.1), the invariant above can be expressed as

$$
T R(K)=\sum_{i, j, k} \operatorname{tr}\left(G^{-2} S\left(f_{j}\right) S^{3}\left(e_{i}\right) S\left(f_{k}\right) e_{j} f_{i} e_{k}\right) .
$$

Except for (1.2), it is clear that there are several other expressions of the cyclic graph $g(\mathcal{L} \cup \mathcal{A})$. For example, if we begin with the vertical line segment $p_{9} \stackrel{x_{9}}{\longrightarrow} p_{10}$, then the expression of cyclic graph $g(\mathcal{L} \cup \mathcal{A})$ is

$$
\bar{p}_{1} \stackrel{\bar{x}_{1}}{\longrightarrow} \bar{p}_{2} \stackrel{\bar{x}_{2}}{\longrightarrow} \ldots \stackrel{\bar{x}_{15}}{\longrightarrow} \bar{p}_{15} \stackrel{\bar{x}_{16}}{\longrightarrow} \bar{p}_{1},
$$

where $\bar{p}_{i}:=p_{i+8}, \bar{x}_{i}:=x_{i+8}$ and $x_{i}=x_{i^{\prime}}$ if $i \equiv i^{\prime} \bmod 16$.

It is easy to see that Kauffman's knot invariant for the trefoil knot $K$ derived from the expression (1.4) of the cyclic graph $g(\mathcal{L} \cup \mathcal{A})$ is

$$
T R(K)=\sum_{i, j, k} \operatorname{tr}\left(G^{-2} S^{4}\left(e_{i}\right) S^{3}\left(f_{j}\right) S^{3}\left(e_{k}\right) S\left(f_{i}\right) S^{2}\left(e_{j}\right) f_{k}\right) .
$$

Using the properties of the trace and (1.1), one can see that (1.3) and (1.5) are the same.

\section{Preliminaries about Hopf algebras}

From this section we will assume that algebras and coalgebras are over a field $k$ with $\operatorname{char}(k) \neq 2$, and $(H, \Delta, \epsilon, S)$ is a finite-dimensional Hopf algebra with the comultiplication $\Delta$, the counit $\epsilon$ and the antipode $S$. Let $H^{*}:=H_{o m}(H, k)$ be the linear dual of $H$. Then $H^{*}$ is also a Hopf algebra. In particular, $H^{*}$ is a $k$-algebra with multiplication

$$
\langle p q, x\rangle=\sum_{(x)}\left\langle p, x_{(1)}\right\rangle\left\langle q, x_{(2)}\right\rangle,
$$

where $p, q \in H^{*}, x \in H$ and $\Delta(x)=\sum_{(x)} x_{(1)} \otimes x_{(2)}$. Note that $H \simeq H^{* *}$ as Hopf algebras. We use $G(H)$ to denote the set consisting of all group-like elements of $H$. Then $G\left(H^{*}\right)=A l g_{k}(H, k)$; that is, $G\left(H^{*}\right)$ consists of all algebra homomorphisms from the algebra $H$ to the algebra $k$.

Definition 2.1. $\quad 1 . \lambda \in H^{*}$ is called a left integral (respectively, right integral) for $H^{*}$ if $h^{*} \lambda=\left\langle h^{*}, 1_{H}\right\rangle \lambda$ (respectively, $\lambda h^{*}=\left\langle h^{*}, 1_{H}\right\rangle \lambda$ ) for every $h^{*} \in H^{*}$.

2. $\Lambda \in H$ is called a left integral (respectively, right integral) for $H$ if $h \Lambda=$ $\epsilon(h) \Lambda$ (respectively, $\Lambda h=\epsilon(h) \Lambda$ ) for every $h \in H$.

It is clear that both left integrals and right integrals form an ideal.

Proposition 2.1. Let $H$ be a finite-dimensional Hopf algebra.

1. Both the ideal of left integrals for $H^{*}$ and the ideal of right integrals for $H^{*}$ are one-dimensional. 
2. $\lambda \in H^{*}$ is a left integral for $H^{*}$ if and only if

$$
\sum_{(h)} h_{(1)} \lambda\left(h_{(2)}\right)=\lambda(h) \quad \text { for } h \in H .
$$

3. $\lambda \in H^{*}$ is a right integral for $H^{*}$ if and only if

$$
\sum_{(h)} \lambda\left(h_{(1)}\right) h_{(2)}=\lambda(h) \quad \text { for } h \in H .
$$

Proof. (1) is a standard result in the theory of finite-dimensional Hopf algebras; see [11] for details.

(2) and (3) follow from (2.1).

Let $\Lambda$ be a non-zero left integral for $H$. By Proposition 2.1, the ideal of left integrals for $H$ is one-dimensional. Hence, there exists a unique $\alpha \in H^{*}$ such that

$$
\Lambda h=\alpha(h) \Lambda \quad \text { for } h \in H,
$$

and $\alpha$ is independent of the choice of $\Lambda$. It follows that $\alpha\left(h h^{\prime}\right)=\alpha(h) \alpha\left(h^{\prime}\right)$ for $h, h^{\prime} \in H$. So $\alpha \in A \lg _{k}(H, k)=G\left(H^{*}\right)$; that is, $\alpha$ is a group-like element of $H^{*}$. Similarly, let $\lambda$ be a non-zero right integral for $H^{*}$, then there exists a unique $g \in \operatorname{Alg}_{k}\left(H^{*}, k\right)=G(H)$ such that

$$
p \lambda=p(g) \lambda \quad \text { for } p \in H^{*} .
$$

Definition 2.2. Let $\Lambda$ be a non-zero left integral for $H$ and $\lambda$ be a non-zero right integral for $H^{*}$. Then the unique group-like element $\alpha$ satisfying (2.4) is called the distinguished group-like element of $H^{*}$, and the unique group-like element $g$ satisfying (2.5) is called the distinguished group-like element of $H$.

$H^{*}$ is an $H$-bimodule under the actions

$$
(h \rightarrow p)(x):=p(x h), \quad(p<h)(x):=p(h x),
$$

and $H$ is a $H^{*}$-bimodule under the actions

$$
p \rightarrow h:=\sum_{(h)} h_{(1)} p\left(h_{(2)}\right), \quad h \leftarrow p:=\sum_{(h)} p\left(h_{(1)}\right) h_{(2)},
$$

where $h, x \in H$ and $p \in H^{*}$.

Definition 2.3. A Hopf algebra $H$ is said to be unimodular if the ideal of left integrals for $H$ is equal to the ideal of right integrals for $H$.

Proposition 2.2. Let $(H, \Delta, \epsilon, S)$ be a finite-dimensional Hopf algebra and $\lambda$ be a non-zero right integral for $H^{*}$. Let $g$ be the distinguished group-like element of $H$ and let $\alpha$ be the distinguished group-like element of $H^{*}$.

1. $\left(H^{*},\llcorner)\right.$ is a free right $H$-module with basis $\lambda .\left(H^{*}, \rightarrow\right)$ is a free left $H$ module with basis $\lambda$.

2. $\lambda(x y)=\lambda\left(S^{2}(y \leftarrow \alpha) x\right)$ for $x, y \in H$.

3. $\lambda(S(x))=\lambda(g x)$ for $x \in H$.

4. Radford's formula holds:

$$
S^{4}(x)=g\left(\alpha \rightarrow x<\alpha^{-1}\right) g^{-1} \quad \text { for } x \in H .
$$

5. $H$ is unimodular if and only if $\alpha=\epsilon$.

6. $H^{*}$ is unimodular if and only if $g=1$.

Proof. The proof of (1)-(4) can be found in [8]. (5) and (6) are clear. 
Proposition 2.3. Let $H$ be a finite-dimensional Hopf algebra. If $H$ is semisimple, then $H$ is unimodular.

Proof. See [5].

Finally, let us recall the definition of a one-sided universal $R$-matrix from [6].

Let $H$ be a Hopf algebra with the comultiplication $\Delta$ and the antipode $S$. Let $\mathcal{R}$ be an element of $H \otimes H$. Consider the following three conditions on $\mathcal{R}$ :
(a) $\mathcal{R} \Delta(x)=\Delta^{\prime}(x) \mathcal{R}$ for all $x \in H$,
(b) $(\Delta \otimes i d)(\mathcal{R})=\mathcal{R}_{13} \mathcal{R}_{23}$,
(c) $(i d \otimes \Delta)(\mathcal{R})=\mathcal{R}_{13} \mathcal{R}_{12}$.

Definition 2.4. In the setting above, we have

1. $\mathcal{R}$ is called an invertible left universal $R-$ matrix if $\mathcal{R}$ is invertible and satisfies (a) and (b).

2. $\mathcal{R}$ is called an invertible right universal $R$-matrix if $\mathcal{R}$ is invertible and satisfies (a) and (c).

3. $\mathcal{R}$ is called a universal $R$-matrix if $\mathcal{R}$ is invertible and satisfies (a), (b) and (c).

4. $\mathcal{R}$ is called an almost cocommutative Hopf algebra if $\mathcal{R}$ is invertible and satisfies (a).

5. $H$ is called a quasi-triangular Hopf algebra if $\mathcal{R}$ is a universal $R$-matrix.

The examples of one-sided invertible $R$-matrices which are not universal $R$ matrices can be found in section 4 .

\section{The TRACE SPACE}

Let $(H, S, \mathcal{R}, G)$ be a quantum algebra. We are interested in the kind of functional $p \in H^{*}$ with the properties

$$
\begin{gathered}
p(x y)=p\left(S^{2 m}(y) x\right) \quad \text { for } x, y \in H, \\
p(x)=p\left(G^{2 m} S(x)\right) \quad \text { for } x \in H,
\end{gathered}
$$

where $m$ is an integer. The set

$$
\mathcal{T}_{m}\left(H^{*}\right):=\left\{p \in H^{*} \mid p \text { satisfies both (3.1) and (3.2) }\right\}
$$

forms a subspace of $H^{*}$. We define $\mathcal{T}_{m}\left(H^{*}\right)$ as the $m$ th trace space for $H$. In particular, $\mathcal{T}_{0}\left(H^{*}\right)$ is called the trace space for $H$, which consists of all traces on $H$.

Proposition 3.1. Let $(H, S, \mathcal{R}, G)$ be a quantum algebra. Then the map $\phi_{m}$ : $\mathcal{T}_{m}\left(H^{*}\right) \stackrel{\sim}{\rightarrow} \mathcal{T}_{0}\left(H^{*}\right)$ given by

$$
\phi_{m}: p \mapsto\left(p<G^{m}\right) \quad \text { for } p \in \mathcal{T}_{m}\left(H^{*}\right)
$$

is a $k$-linear isomorphism.

Proof. Use (3.1) and (3.2).

Definition 3.1. A quantum algebra $(H, S, \mathcal{R}, G)$ is said to be a quantum Hopf algebra if $H$ is a Hopf algebra with the antipode $S$. A quantum Hopf algebra is denoted by $(H, \Delta, \epsilon, S, \mathcal{R}, G)$, where $(H, \Delta, \epsilon, S)$ gives the Hopf algebra structure on $H$ and $(H, S, \mathcal{R}, G)$ gives the quantum algebra structure on $H$. 
An interesting behaviour of the trace space $\mathcal{T}_{0}\left(H^{*}\right)$ for a quantum Hopf algebra $H$ is that $\mathcal{T}_{0}\left(H^{*}\right)$ is a Jordan subalgebra of $\left(H^{*}\right)^{+}$, where $\left(H^{*}\right)^{+}$is the Jordan algebra obtained from the associative algebra $H^{*}$. In general, we have

Proposition 3.2. Let $(H, \Delta, \epsilon, S, \mathcal{R}, G)$ be a quantum Hopf algebra.

1. $\mathcal{T}_{m}\left(H^{*}\right)$ is a Jordan subalgebra of $\left(H^{*}\right)^{+}$if $G^{2 m} \in G(H)$.

2. $\mathcal{T}_{m}\left(H^{*}\right)$ is a commutative subalgebra of $H^{*}$ if $G^{2 m} \in G(H)$ and $H$ is almost cocommutative.

3. The map $\phi_{m}: \mathcal{T}_{m}\left(H^{*}\right) \stackrel{\sim}{\rightarrow} \mathcal{T}_{0}\left(H^{*}\right)$ is a Jordan isomorphism if $G^{m} \in G(H)$.

Proof. Let $m: k \otimes k \rightarrow k$ be the multiplication in $k$. Then the multiplication (2.1) in $H^{*}$ can be expressed as

$$
(p q)(x)=m(p \otimes q) \Delta(x) \quad \text { for } x \in H \text { and } p, q \in H^{*} .
$$

(1) Let $p, q \in T_{m}\left(H^{*}\right)$ and $x, y \in H$. By (3.1), we have

$$
\begin{aligned}
(p \otimes q) \Delta(x y) & =(p \otimes q)\left\{\left[\left(S^{2 m} \otimes S^{2 m}\right) \Delta(y)\right] \Delta(x)\right\} \\
& =(p \otimes q)\left\{\Delta\left(S^{2 m}(y)\right) \Delta(x)\right\}=(p \otimes q) \Delta\left(S^{2 m}(y) x\right),
\end{aligned}
$$

which implies that

$$
(p q)(x y)=(p q)\left(S^{2 m}(y) x\right) .
$$

Similarly, it follows from (3.2) that

$$
\begin{aligned}
(p \otimes q) \Delta(x) & =(p \otimes q)\left\{\left(G^{2 m} \otimes G^{2 m}\right)[(S \otimes S) \Delta(x)]\right\} \\
& =(p \otimes q)\left\{\left(G^{2 m} \otimes G^{2 m}\right) \sigma \Delta(S(x))\right\} \\
& =(p \otimes q) \sigma\left\{\Delta\left(G^{2 m}\right) \Delta(S(x))\right\}=(q \otimes p) \Delta\left(G^{2 m} S(x)\right),
\end{aligned}
$$

which implies that

$$
(p q)(x)=(q p)\left(G^{2 m} S(x)\right) .
$$

From (3.3) and (3.4), we know that if $p, q \in T_{m}\left(H^{*}\right)$, then $\frac{1}{2}(p q+q p) \in T_{m}\left(H^{*}\right)$. This proves (1).

(2) Let $p, q \in T_{m}\left(H^{*}\right)$ and $x \in H$. Using (1.1), we get

$$
\begin{aligned}
(p \otimes q) \Delta(x) & =(q \otimes p) \Delta^{\prime}(x)=(q \otimes p)\left\{\mathcal{R} \Delta(x) \mathcal{R}^{-1}\right\} \\
& =(q \otimes p)\left\{\left(S^{2 m} \otimes S^{2 m}\right)\left(\mathcal{R}^{-1}\right) \mathcal{R} \Delta(x)\right\} \\
& =(q \otimes p)\left\{\mathcal{R}^{-1} \mathcal{R} \Delta(x)\right\}=(q \otimes p) \Delta(x) .
\end{aligned}
$$

This proves that $p q=q p$. Hence, $T_{m}\left(H^{*}\right)$ is a commutative subalgebra of $H^{*}$ by (3.3) and (3.4).

(3) This fact follows from the following equation:

$$
(p q)\left\llcorner a=\left(p \llcorner a ) \left(q\llcorner a) \quad \text { for } p, q \in H^{*} \text { and } a \in G(H) .\right.\right.\right.
$$

Let $H$ be a finite-dimensional Hopf algebra. By Proposition 2.2, we have a $k$-linear isomorphism $\psi: H^{*} \stackrel{\sim}{\rightarrow} H$ defined by

$$
\psi:(\lambda<h) \mapsto h \quad \text { for } h \in H,
$$

where $\lambda$ is a non-zero right integral for $H^{*}$. 
If $H$ is a finite-dimensional quantum Hopf algebra, we set

$$
r_{m}(H):=\psi\left(T_{m}\left(H^{*}\right)\right) .
$$

The vector space $r_{m}(H)$ is called the $m$ th right counterpart of the trace space and can be described as follows.

Proposition 3.3. Let $(H, S, \mathcal{R}, G)$ be a finite-dimensional quantum Hopf algebra and $\lambda$ be a non-zero right integral for $H^{*}$. Let $g$ be the distinguished group-like element of $H$ and let $\alpha$ be the distinguished group-like element of $H^{*}$.

1. Suppose that $p=\lambda \leftarrow h$ for some $h \in H$. Then

(a) $p$ satisfies (3.1) if and only if $h$ satisfies

$$
S^{2}(x<\alpha) h=h S^{2 m}(x) \quad \text { for } x \in H .
$$

(b) $p$ satisfies (3.2) if and only if $h$ satisfies

$$
\text { 2. } r_{m}(H)=\{h \in H \mid \quad h \text { satisfies both (3.5) and (3.6) }\} \text {. }
$$

Proof. (1) Let us prove (b). The proof of (a) is similar.

For any $x \in H$, by Proposition 2.2 (2) and (3), we have

$$
\begin{aligned}
p\left(G^{2 m} S(x)\right) & =(\lambda \leftarrow h)\left(G^{2 m} S(x)\right)=\lambda\left(h G^{2 m} S(x)\right) \\
& =(\lambda S)\left(x G^{-2 m} S^{-1}(h)\right)=\lambda\left(g x G^{-2 m} S^{-1}(h)\right) \\
& =\lambda\left(S^{2}\left(\left[G^{-2 m} S^{-1}(h)\right] \leftarrow \alpha\right) g x\right)=(x \rightarrow \lambda)\left(S^{2}\left(\left[G^{-2 m} S^{-1}(h)\right] \leftarrow \alpha\right) g\right)
\end{aligned}
$$

or

$$
p\left(G^{2 m} S(x)\right)=(x-\lambda)\left(S^{2}\left(\left[G^{-2 m} S^{-1}(h)\right] \leftarrow \alpha\right) g\right) \quad \text { for } x \in H .
$$

It is clear that

$$
p(x)=(x \rightarrow \lambda)(h) \quad \text { for } x \in H .
$$

It follows from (3.7) and (3.8) that $p$ satisfies (3.2) if and only if

$$
(x \rightarrow \lambda)\left(S^{2}\left(\left[G^{-2 m} S^{-1}(h)\right]<\alpha\right) g\right)=(x-\lambda)(h) \quad \text { for } x \in H,
$$

which is equivalent to saying that $h$ satisfies (3.6).

(2) Use (1) and the definition of $r_{m}(H)$.

Proposition 3.4. If $(H, S, \mathcal{R}, G)$ is a unimodular finite-dimensional quantum Hopf algebra, then $G^{2} g^{-1} \in Z(H)$ and

$$
r_{m}(H)=\left\{h \in H \mid \quad h=z G^{1-m} \text { and } S(z)=z G^{2} g^{-1} \text { for some } z \in Z(H)\right\}
$$

where $g$ is the distinguished group-like element of $H$.

Proof. Since $H$ is unimodular, $\alpha=\epsilon$ by Proposition 2.2 (5). Using Radford's formula in Proposition 2.2, we see that $G^{2} g^{-1} \in Z(H)$, where $Z(H)$ is the center of $H$. Also, (3.5) becomes $S^{2}(x) h=h S^{2 m}(x)$ for $x \in H$. Hence, $h G^{m-1}=z \in Z(H)$ or $h=z G^{1-m}$.

By (3.6), $G^{-2 m} S(h)=h g^{-1}$ or $G^{-2 m} S\left(z G^{1-m}\right)=z G^{1-m} g^{-1}$, which implies that

$$
S(z)=z G^{2} g^{-1}, \quad z \in Z(H) .
$$


Remark. If $(H, \mathcal{R})$ is a quasi-triangular Hopf algebra, then the description of $r_{0}(H)$ is equivalent to the description of $\operatorname{Cocom}_{S}\left(H^{*}\right)$ given in [7].

Now we can determine the trace space for a semisimple finite-dimensional quantum Hopf algebra.

Proposition 3.5. Let $(H, S, \mathcal{R}, G)$ be a semisimple finite-dimensional quantum Hopf algebra over an algebraically close field $k$. Let $g$ be the distinguished group-like element of $H$.

1. $Z(H)=k e_{1} \oplus \cdots \oplus k e_{n}$, where $e_{1}, \ldots, e_{n}$ are pairwise orthogonal idempotents.

2. Let $G^{2} g^{-1}=d_{1} e_{1}+\cdots+d_{n} e_{n}$, where $d_{i} \in k$. Then there exists a permutation $\tau$ of $\{1,2, \ldots, n\}$ such that $S\left(e_{i}\right)=e_{\tau(i)}$ and the set

$$
\left\{\left(d_{\tau(i)} e_{i}+e_{\tau(i)}\right) G^{1-m} \mid d_{i} d_{\tau(i)}=1, \quad d_{\tau(i)} \neq-1 \quad \text { for some } 1 \leq i \leq n\right\}
$$

is a $k$-linear basis of $r_{m}(H)$.

Proof. (1) This is a standard result in the theory of finite-dimensional associative algebras.

(2) Since the center $Z(H)$ is invariant under the antipode $S$, we know that $S\left(e_{i}\right)$ is an idempotent in the center $Z(H)$. Hence, $S\left(e_{i}\right)=e_{i 1}+\cdots+e_{i k_{i}}$, where $k_{i} \geq 1$ and $\left\{e_{i 1}, \ldots, e_{i k_{i}}\right\}$ is a subset of $\left\{e_{1}, \ldots, e_{n}\right\}$. By $e_{i} e_{j}=0$ for $i \neq j$, we get

$$
\left\{e_{i 1}, \ldots, e_{i k_{i}}\right\} \cap\left\{e_{j 1}, \ldots, e_{j k_{j}}\right\}=\emptyset \quad \text { for } i \neq j .
$$

This proves that $k_{i}=1$ for every $1 \leq i \leq n$.

Let $S\left(e_{i}\right)=e_{\tau(i)}$. Then $\tau$ is a permutation of $\{1,2, \ldots, n\}$. That $S^{2}$ is inner implies that $\tau^{2}=1$.

By Proposition 2.3, $H$ is unimodular. Hence, $r_{m}(H)$ is described by Proposition 3.4. Let $z=x_{1} e_{1}+\cdots+x_{n} e_{n} \in Z(H)$, where $x_{i} \in k$. Then, (3.9) holds if and only if $x_{i}=x_{\tau(i)} d_{\tau(i)}$ for $1 \leq i \leq n$, which implies that

$$
0= \begin{cases}x_{i}\left(1-d_{i} d_{\tau(i)}\right), & \text { if } \tau(i) \neq i, \\ x_{i}\left(1-d_{i}\right), & \text { if } \tau(i)=i .\end{cases}
$$

This proves that $x_{i}=0$ if either $d_{i} d_{\tau(i)} \neq 1$ and $\tau(i) \neq i$ or $d_{i} \neq 1$ and $\tau(i)=i$. In other words, $z G^{1-m}$ is a linear combination of the elements of the set in (2). Therefore, the set is a $k$-basis of $r_{m}(H)$ because it is clearly linearly independent.

Now we present the best lower bound for the dimension of the trace space.

Proposition 3.6. Let $(H, S, \mathcal{R}, G)$ be a unimodular finite-dimensional quantum Hopf algebra, and let $g$ be the distinguished group-like element of $H$. Then the best lower bound of $\operatorname{dim} \mathcal{T}_{0}\left(H^{*}\right)$ is

$$
\max \left\{\operatorname{dim} \operatorname{ker}\left(\left(S-L_{G^{2} g^{-1}}\right) \mid A\right) \mid A \in W(H)\right\},
$$

where $L_{G^{2} g^{-1}}: A \rightarrow A$ is the multiplication given by $G^{2} g^{-1}$ and

$$
W(H):=\left\{A \mid A \text { is a subalgebra of } Z(H) \text { such that } G^{2} g^{-1} \in A \text { and } S(A)=A\right\} \text {. }
$$

Proof. By Proposition 3.4, $Z(H) \in W(H)$. Hence, $W(H)$ is not empty. Consider any element $A$ in $W(H)$. Since $G^{2} g^{-1} \in A$ and $S(A)=A,\left(S-L_{G^{2} g^{-1}}\right) \mid A$ is a well-defined linear map on $A$. Using Proposition 3.4 again, we have

$$
h \in r_{1}(H) \cap A \Longleftrightarrow S(h)=h G^{2} g^{-1} \text { and } h \in A \Longleftrightarrow h \in \operatorname{ker}\left(\left(S-L_{G^{2} g^{-1}}\right) \mid A\right) \text {. }
$$


It follows that $r_{1}(H) \cap A=\operatorname{ker}\left(\left(S-L_{G^{2} g^{-1}}\right) \mid A\right)$. Therefore,

$$
\begin{aligned}
\operatorname{dim} \mathcal{T}_{0}\left(H^{*}\right)=\operatorname{dim} r_{1}(H) & \geq \operatorname{dim}\left(r_{1}(H) \cap A\right) \\
& =\operatorname{dim} \operatorname{ker}\left(\left(S-L_{G^{2} g^{-1}}\right) \mid A\right) \quad \text { for } A \in W(H) .
\end{aligned}
$$

This proves that $\max \left\{\operatorname{dim} \operatorname{ker}\left(\left(S-L_{G^{2} g^{-1}}\right) \mid A\right) \mid A \in W(H)\right\}$ is a lower bound of $\operatorname{dim} \mathcal{T}_{0}\left(H^{*}\right)$.

In order to prove that the lower bound above is the best one, we will study a class of unimodular finite-dimensional quantum Hopf algebras $s \ell_{q, z}^{t}(2)$ in $\S 4$ and prove that $\operatorname{dim} \mathcal{T}_{0}\left(s \ell_{q, z}^{t}(2)^{*}\right)$ is equal to the lower bound above for odd $t$ in $\S 5$.

We finish this section by calculating $\operatorname{dim} \operatorname{ker}\left(\left(S-L_{G^{2} g^{-1}}\right) \mid A\right)$ for some special algebras in $W(H)$.

Proposition 3.7. Let $(H, S, \mathcal{R}, G)$ be a unimodular finite-dimensional quantum Hopf algebra, and let $g$ be the distinguished group-like element of $H$. If $P:=$ $\left\{z_{1}, z_{2}, \ldots, z_{n}\right\}$ is a subgroup of $Z(H) \cap G(H)$ such that $G^{2} g^{-1} \in k[P]$, where $k[P]$ is the group algebra of the group $P$, then

1. There exists a $\tau \in \mathbb{S}_{n}$ such that $S\left(z_{i}\right)=z_{\tau(i)}$ for $1 \leq i \leq n$ and $\tau^{2}=1$, where $\mathbb{S}_{n}$ is the symmetric group on the set $\{1,2, \ldots, n\}$.

2. $\operatorname{dim} \operatorname{ker}\left(\left(S-L_{G^{2} g^{-1}}\right) \mid k[P]\right)=n-\operatorname{rank}\left(\left(d_{\tau \sigma_{i}(j)}\right)_{1 \leq i, j \leq n}-I_{n \times n}\right)$, where $G^{2} g^{-1}:=d_{1} z_{1}+\ldots d_{n} z_{n}, d_{i} \in k, I_{n \times n}$ is the identity matrix and the map $z_{i} \mapsto \sigma_{i}$ is the regular representation of the group $P$ in the symmetric group $\mathbb{S}_{n}$

Proof. (1) The existence of $\tau$ follows from the fact that $S(h)=h^{-1}$ for $h \in G(H)$.

(2) Let $h:=x_{1} z_{1}+\ldots x_{n} z_{n} \in k[P]$, where $x_{i} \in k$. We then have that $h \in$ $\operatorname{ker}\left(\left(S-L_{G^{2} g^{-1}}\right) \mid k[P]\right)$ if and only if $S(h)=h G^{2} g^{-1}$ and $h \in k[P]$, which is equivalent to

$$
\sum_{i=1}^{n} d_{\tau \sigma_{i}(j)} x_{i}=x_{j} \quad \text { for } 1 \leq i \leq n
$$

This proves that the vector space $\operatorname{ker}\left(\left(S-L_{G^{2} g^{-1}}\right) \mid k[P]\right)$ is isomorphic to the solution space of the homogeneous linear system (3.10). So (2) is true.

Corollary 3.8. Let $(H, S, \mathcal{R}, G)$ be a unimodular finite-dimensional quantum Hopf algebra. Let $g$ be the distinguished group-like element of $H$. If $P:=\left\{1, z, \ldots, z^{n-1}\right\}$ is a cyclic subgroup of $Z(H) \cap G(H)$ with the order $n$ and $G^{2} g^{-1}=z^{m}$ for some $m \in \mathbb{Z}$ and $0 \leq m \leq n-1$, then

$$
\operatorname{dim} \operatorname{ker}\left(\left(S-L_{G^{2} g^{-1}}\right) \mid k[P]\right)= \begin{cases}\frac{n+2}{2}, & \text { if both } n \text { and } m \text { are even, } \\ {\left[\frac{n+1}{2}\right],} & \text { otherwise, }\end{cases}
$$

where $[x]$ is the largest integer that is less than or equal to $x$.

Proof. From the proof of Proposition 3.7 above, we know that $h=\sum_{i=0}^{n-1} x_{i} z^{i} \in$ $\operatorname{ker}\left(\left(S-L_{G^{2} g^{-1}}\right) \mid k[P]\right)$ if and only if (3.10) holds. Under our assumption, (3.10) becomes

$$
x_{-i}=x_{i-m} \quad \text { for } 0 \leq i \leq n-1 .
$$

Consequently, every $h \in \operatorname{ker}\left(\left(S-L_{G^{2} g^{-1}}\right) \mid k[P]\right)$ is a linear combination of the elements in the set $\left\{z^{-i}+z^{i-m} \mid \quad 0 \leq i \leq n-1\right\}$. This implies that a maximal linearly independent subset of the set $\left\{z^{-i}+z^{i-m} \mid 0 \leq i \leq n-1\right\}$ is a basis of 
$\operatorname{ker}\left(\left(S-L_{G^{2} g^{-1}}\right) \mid k[P]\right)$. Hence, $\operatorname{dim} \operatorname{ker}\left(\left(S-L_{G^{2} g^{-1}}\right) \mid k[P]\right)$ is the number of elements in a maximal linearly independent subset of the set $\left\{z^{-i}+z^{i-m} \mid 0 \leq i \leq\right.$ $n-1\}$. One can check that the number is either $\frac{n+2}{2}$ or $\left[\frac{n+1}{2}\right]$.

For example, if both $n$ and $m$ are odd, then there exist $m_{1}, \ldots, m_{\frac{n-1}{2}} \in\{0,1, \ldots$, $n-1\}$ such that

$$
\left\{-\frac{m+n}{2},-m_{1}, m_{1}-m, \ldots,-m_{\frac{n-1}{2}}, m_{\frac{n-1}{2}}-m\right\}
$$

is a complete residue system modulo $n$. Hence

$$
\left\{z^{-\frac{m+n}{2}}, z^{-m_{1}}+z^{m_{1}-m}, \ldots, z^{-m_{\frac{n-1}{2}}}+z^{m_{\frac{n-1}{2}}-m}\right\}
$$

is the maximal linearly independent subset of the set $\left\{z^{-i}+z^{i-m} \mid 0 \leq i \leq n-1\right\}$. So $\operatorname{dim} \operatorname{ker}\left(\left(S-L_{G^{2} g^{-1}}\right) \mid k[P]\right)=\frac{n-1}{2}+1=\frac{n+1}{2}=\left[\frac{n+1}{2}\right]$.

\section{The Quantum Hopf Algebra $s \ell_{q, z}^{t}(2)$}

Throughout this section and section 5 , we assume that $q^{2}$ is a $t$ th primitive root of unity with $t \geq 2$. Recall from [6] that $s \ell_{q, z}^{t}(2)$ is a finite-dimensional Hopf algebra over $\mathbb{C}$ with generators $\left\{E, F, K^{ \pm 1}, z^{ \pm 1}\right\}$ and relations

$$
\begin{aligned}
& z z^{-1}=z^{-1} z=1 \text { and } z \text { is in the center of } s \ell_{q, z}^{t}(2), \\
& K K^{-1}=K^{-1} K=1, \\
& K E K^{-1}=q^{2} E, \quad K F K^{-1}=q^{-2} F, \\
& E F-F E=\frac{K-z^{-1} K^{-1}}{q-q^{-1}}, \\
& E^{t}=F^{t}=0, \quad K^{t}=z^{t}=1,
\end{aligned}
$$

where the comultiplication $\Delta$, the counit $\epsilon$ and the antipode $S$ are defined by

$$
\begin{array}{llrl}
\Delta(E)=E \otimes 1+K \otimes E, & \epsilon(E)=0, & S(E)=-K^{-1} E, \\
\Delta(F)=F \otimes z^{-1} K^{-1}+1 \otimes F, & \epsilon(F)=0, & S(F)=-F z K, \\
\Delta(X)=X \otimes X, & \epsilon(X)=1, & S(X)=X^{-1}
\end{array}
$$

for $X \in\left\{K^{ \pm 1}, z^{ \pm 1}\right\}$.

We define two varieties $\bar{V}_{\ell}$ and $\bar{V}_{r}$ in $\mathbb{C}^{t^{2}}$ as follows:

$$
\begin{aligned}
& V_{\ell}:=\left\{\left(d_{\alpha \beta}\right)_{0 \leq \alpha, \beta<t} \mid \sum_{0 \leq \alpha<t} q^{2\left(\gamma^{\prime}-\gamma\right) \alpha} d_{\alpha, \gamma} d_{\beta-\alpha, \gamma^{\prime}}=\delta_{\gamma, \gamma^{\prime}} d_{\beta, \gamma}\right\}, \\
& V_{r}:=\left\{\left(d_{\alpha \beta}\right)_{0 \leq \alpha, \beta<t} \mid \sum_{0 \leq \alpha<t} q^{2\left(\beta^{\prime}-\beta\right) \alpha} d_{\beta, \alpha} d_{\beta^{\prime}, \gamma-\alpha}=\delta_{\beta, \beta^{\prime}} d_{\beta, \gamma}\right\}, \\
& \bar{V}_{\ell}:=\left\{\left(d_{\alpha \beta}\right)_{0 \leq \alpha, \beta<t} \in V_{\ell} \mid \sum_{0 \leq \beta, \gamma<t} q^{-2 b \beta+2 d \gamma} d_{\beta \gamma} d_{-\beta-d, \gamma-b}=\delta_{(b, d),(0,0)}\right\}, \\
& \bar{V}_{r}:=\left\{\left(d_{\alpha \beta}\right)_{0 \leq \alpha, \beta<t} \in V_{r} \mid \sum_{0 \leq \beta, \gamma<t} q^{-2 b \beta+2 d \gamma} d_{\beta \gamma} d_{-\beta-d, \gamma-b}=\delta_{(b, d),(0,0)}\right\},
\end{aligned}
$$

where $\gamma, \gamma^{\prime}, b, d \in\{0,1, \ldots, t-1\}$ and $d_{\alpha, \beta}=d_{\alpha^{\prime}, \beta^{\prime}}$ if $\alpha \equiv \alpha^{\prime} \bmod t$ and $\beta \equiv \beta^{\prime}$ $\bmod t$. 
Note that the variety $\bar{V}_{\ell} \cap \bar{V}_{r}$ contains at least the following symmetric points:

$$
\left(x_{\alpha, \beta}\right)_{0 \leq \alpha, \beta<t}=\left(\begin{array}{cccc}
1 & 0 & \ldots & 0 \\
0 & 0 & \ldots & 0 \\
\vdots & \vdots & \ddots & \vdots \\
0 & 0 & \ldots & 0
\end{array}\right)
$$

and

$$
\left(x_{\alpha, \beta}\right)_{0 \leq \alpha, \beta<t}=\frac{1}{t}\left(\begin{array}{cccc}
1 & 1 & \ldots & 1 \\
1 & a & \ldots & a^{t-1} \\
\vdots & \vdots & \ddots & \vdots \\
1 & a^{t-1} & \ldots & a^{(t-1)^{2}}
\end{array}\right),
$$

where $a \in \mathbb{C}, a^{t}=1,\langle a\rangle=\left\langle q^{2}\right\rangle$ and $a \neq q^{2}$.

For $m \in \mathbb{Z}$, we define

$$
\begin{aligned}
& {[m]:=\frac{q^{m}-q^{-m}}{q-q^{-1}}, \quad[m] !:=[m][m-1] \ldots[1],} \\
& \left(\begin{array}{c}
m \\
n
\end{array}\right):= \begin{cases}\frac{[m] !}{[n] ![m-n] !}, & \text { for } 1 \leq n<m, \\
1, & \text { for } n=0 \text { or } n=m,\end{cases} \\
& {[K, z ; m]:=\frac{q^{m} K-q^{-m} z^{-1} K^{-1}}{q-q^{-1}},} \\
& \left(\begin{array}{ccc}
K, & z ; & m \\
& j &
\end{array}\right):=\frac{[K, z ; m][K, z ; m-1] \ldots[K, z ; m-j+1]}{[j] !} .
\end{aligned}
$$

Lemma 4.1. In $s \ell_{q, z}^{t}(2)$, we have

$$
\begin{aligned}
& F^{n} E^{k}=\sum_{0 \leq i \leq \min (n, k)}(-1)^{i}\left(\begin{array}{c}
n \\
i
\end{array}\right)[i] !\left(\begin{array}{c}
k \\
i
\end{array}\right)[i] !\left(\begin{array}{ccc}
K, & z ; & n-k-1+i \\
i
\end{array}\right) E^{k-i} F^{n-i}, \\
& (3.1) \quad F^{n} E^{n}=\sum_{0 \leq j \leq i \leq n}(-1)^{i}\left(\begin{array}{c}
n \\
i
\end{array}\right)^{2}[i] ! \frac{q^{\frac{(2 j-i)(i-1)}{2}}}{\left(q-q^{-1}\right)^{i}}\left(\begin{array}{c}
i \\
j
\end{array}\right) E^{n-i} F^{n-i} K^{2 j-i} z^{j-i} .
\end{aligned}
$$

Proof. The proof is similar to the proof given in [4]. See [4] for details.

Proposition 4.2. Let

$$
\begin{aligned}
& \mathcal{R}_{\left(d_{\alpha \beta}\right)_{0 \leq \alpha, \beta<t}} \\
& =\frac{1}{t} \sum_{0 \leq n, a, b, \gamma, \mu<t} \frac{\left(q^{-1}-q\right)^{n}}{[n] !} q^{2 a b+2 \mu \gamma-2 b \gamma+\frac{n(1-n)}{2}} d_{b-\mu, \gamma} E^{n} K^{a} z^{\gamma} \otimes F^{n} K^{b} z^{\mu},
\end{aligned}
$$

where $d_{\alpha, \beta} \in \mathbb{C}$. Then

1. $\left(s \ell_{q, z}^{t}(2), S, \mathcal{R}_{\left(d_{\alpha \beta}\right)_{0 \leq \alpha, \beta<t}}, K^{-1} z^{-1-\xi}\right)$ is a quantum Hopf algebra if

$$
\left(d_{\alpha, \beta}\right)_{0 \leq \alpha, \beta<t} \in \bar{V}_{\ell} \cup \bar{V}_{r} \text { and } \xi \in \mathbb{Z} / t \mathbb{Z} .
$$

2. $\left(s \ell_{q, z}^{t}(2), \mathcal{R}_{\left(d_{\alpha \beta}\right)_{0 \leq \alpha, \beta<t}}\right)$ is a quasi-triangular Hopf algebra if and only if $\left(d_{\alpha, \beta}\right)_{0 \leq \alpha, \beta<t}$ is in $\bar{V}_{r} \cap \bar{V}_{r}$. 
Proof. (1) By Theorem 2.1 in [6], if $\left(d_{\alpha, \beta}\right)_{0 \leq \alpha, \beta<t} \in \bar{V}_{\ell} \cup \bar{V}_{r}$, then $\mathcal{R}_{\left(d_{\alpha \beta}\right)_{0 \leq \alpha, \beta<t}}$ is a one-sided invertible universal $R$-matrix satisfying the conditions (2) and (3) in Definition 1.1. It is clear that the condition (4) holds for $G:=K^{-1} z^{-1-\xi}$, where $\xi \in \mathbb{Z} / t \mathbb{Z}$.

(2) See Theorem 2.1 in [6].

Since the set $\left(\bar{V}_{\ell} \cup \bar{V}_{r}\right) \backslash\left(\bar{V}_{r} \cap \bar{V}_{r}\right)$ is far from empty, the examples of quantum Hopf algebras are richer than those of quasi-triangular Hopf algebras.

For example, if $t=2$, then

$$
\begin{aligned}
& \bar{V}_{\ell} \cup \bar{V}_{r}=\left\{\left(\begin{array}{ll}
1 & 0 \\
0 & 0
\end{array}\right),\left(\begin{array}{ll}
0 & 1 \\
0 & 0
\end{array}\right),\left(\begin{array}{ll}
0 & 0 \\
1 & 0
\end{array}\right),\left(\begin{array}{cc}
\frac{1}{2} & \frac{1}{2} \\
\frac{1}{2} & \frac{1}{2}
\end{array}\right),\left(\begin{array}{cc}
\frac{1}{2} & \frac{1}{2} \\
-\frac{1}{2} & -\frac{1}{2}
\end{array}\right),\left(\begin{array}{cc}
\frac{1}{2} & -\frac{1}{2} \\
\frac{1}{2} & -\frac{1}{2}
\end{array}\right)\right\}, \\
& \bar{V}_{\ell} \cap \bar{V}_{r}=\left\{\left(\begin{array}{ll}
1 & 0 \\
0 & 0
\end{array}\right),\left(\begin{array}{cc}
\frac{1}{2} & \frac{1}{2} \\
\frac{1}{2} & \frac{1}{2}
\end{array}\right)\right\} .
\end{aligned}
$$

Proposition 4.3. 1. The set $\left\{E^{\alpha} F^{\beta} K^{\gamma} z^{\delta} \mid \quad 0 \leq \alpha, \beta, \gamma, \delta<t\right\}$ is a $\mathbb{C}$-linear basis for $s \ell_{q, z}^{t}(2)$.

2. For $0 \leq \alpha, \beta, \gamma, \delta<t$, we have

$$
\begin{aligned}
& \Delta\left(E^{\alpha} F^{\beta} K^{\gamma} z^{\delta}\right) \\
= & \sum_{\substack{0 \leq k \leq \alpha \\
0 \leq \ell \leq \beta}} q^{k(\alpha-k)+(\beta-\ell)(\ell-2 k)}\left(\begin{array}{l}
\alpha \\
k
\end{array}\right)\left(\begin{array}{l}
\beta \\
\ell
\end{array}\right) E^{\alpha-k} F^{\beta-\ell} K^{\gamma+k} z^{\delta} \otimes E^{k} F^{\ell} K^{\gamma-\beta+\ell} z^{\delta-\beta+\ell} .
\end{aligned}
$$

Proof. (1) follows from an application of the Diamond Lemma for rings. (2) follows from the $q$-analogue of the Binomial Theorem:

$$
(A+B)^{n}=\sum_{k=0}^{n} q^{k^{2}-n k}\left(\begin{array}{l}
n \\
k
\end{array}\right) B^{k} A^{n-k}, \quad \text { where } B A=q^{2} A B .
$$

For a non-zero element $a$ in $s \ell_{q, z}^{t}(2)$, we use $\bar{a}$ to denote the functional defined by

$$
\bar{a}(x)= \begin{cases}1, & \text { if } x=a \\ 0, & \text { if } x \neq a .\end{cases}
$$

Proposition 4.4. 1. The Hopf algebra $s \ell_{q, z}^{t}(2)$ is unimodular.

2. $\Lambda=\left(\sum_{0 \leq \alpha, \beta<t} K^{\alpha} z^{\beta}\right) E^{t-1} F^{t-1}$ is a non-zero two-sided integral for $s \ell_{q, z}^{t}(2)$.

3. $\overline{E^{t-1} F^{t-1} K^{t-1} z^{t-1}}$ is a non-zero right integral for $s \ell_{q, z}^{t}(2)^{*}$.

4. $\overline{E^{t-1} F^{t-1} K}$ is a non-zero left integral for $s \ell_{q, z}^{t}(2)^{*}$.

5. The distinguished group-like element of $s \ell_{q, z}^{t}(2)$ is $g=K^{t-2} z^{t-1}$.

Proof. (1) and (2). Let $\Lambda$ be a non-zero left integral for $s \ell_{q, z}^{t}(2)$. Let $\alpha$ be the distinguished group-like element of $s \ell_{q, z}^{t}(2)^{*}$. Then

$$
h \Lambda=\epsilon(h) \Lambda, \quad \Lambda h=\alpha(h) \Lambda \quad \text { for } h \in s \ell_{q, z}^{t}(2) .
$$

Taking $h$ to be $E^{t}$ and $F^{t}$ in (4.1) respectively, we get

$$
\epsilon(E)=\alpha(E)=0, \quad \epsilon(F)=\alpha(F)=0 .
$$


Let $\Lambda=\sum_{\substack{\alpha_{0} \leq \alpha<t, \beta_{0} \leq \beta<t}} E^{\alpha} F^{\beta} a_{\alpha, \beta}$, where $a_{\alpha, \beta} \in \mathbb{C}\left[z^{ \pm 1}, K^{ \pm 1}\right]$ and $a_{\alpha_{0}, \beta_{0}} \neq 0$. Taking $h$ to be $E$ and $F$ in (4.1) respectively, we get that $\alpha_{0}=\beta_{0}=t-1$. Hence, $\Lambda=E^{t-1} F^{t-1} a$ for some $a \in \mathbb{C}\left[z^{ \pm 1}, K^{ \pm 1}\right]$.

Letting $h$ be any $X \in\left\{z^{ \pm 1}, K^{ \pm 1}\right\}$ in (4.1), we get $X a=a$, which implies that $a$ is a scalar multiple of $\sum_{0 \leq \alpha, \beta<t} K^{\alpha} z^{\beta}$. This proves that

$$
\Lambda=E^{t-1} F^{t-1}\left(\sum_{0 \leq \alpha, \beta<t} K^{\alpha} z^{\beta}\right)=\left(\sum_{0 \leq \alpha, \beta<t} K^{\alpha} z^{\beta}\right) E^{t-1} F^{t-1}
$$

is a non-zero left integral for $s \ell_{q, z}^{t}(2)$.

It is clear that $\Lambda=\Lambda X=\alpha(X) \Lambda$ for $X \in\left\{z^{ \pm 1}, K^{ \pm 1}\right\}$. Hence,

$$
\alpha(X)=1=\epsilon(X) \quad \text { for } X \in\left\{z^{ \pm 1}, K^{ \pm 1}\right\} .
$$

It follows from (4.2) and (4.3) that $\alpha=\epsilon$; that is, $s \ell_{q, z}^{t}(2)$ is unimodular. So $\Lambda=\left(\sum_{0 \leq \alpha, \beta<t} K^{\alpha} z^{\beta}\right) E^{t-1} F^{t-1}$ is a non-zero two-sided integral for $s \ell_{q, z}^{t}(2)$.

(3) Let $\lambda$ be a non-zero right integral for $s \ell_{q, z}^{t}(2)^{*}$. By (2.3) and Proposition $4.3(2)$, we have

$$
\lambda\left(E^{\alpha} F^{\beta} K^{\gamma} z^{\delta}\right)=\sum_{\substack{0 \leq k \leq \alpha \\ 0 \leq \ell \leq \beta}} A_{\alpha \beta k \ell} \lambda\left(E^{\alpha-k} F^{\beta-\ell} K^{\gamma+k} z^{\delta}\right) E^{k} F^{\ell} K^{\gamma-\beta+\ell} z^{\delta-\beta+\ell}
$$

where $0 \leq \alpha, \beta, \gamma, \delta<t$ and

$$
A_{\alpha \beta k \ell}:=q^{k(\alpha-k)+(\beta-\ell)(\ell-2 k)}\left(\begin{array}{l}
\alpha \\
k
\end{array}\right)\left(\begin{array}{l}
\beta \\
\ell
\end{array}\right) \neq 0
$$

for $0 \leq k \leq \alpha(<t)$ and $0 \leq \ell \leq \beta(<t)$.

Since $\lambda\left(E^{\alpha} F^{\beta} K^{\gamma} z^{\delta}\right) \in \mathbb{C} 1_{H}$, we conclude from (4.4) that if $\lambda\left(E^{\alpha} F^{\beta} K^{\gamma} z^{\delta}\right) \neq 0$, then $\beta=\gamma=\delta$.

Now we assume that $\lambda\left(E^{\alpha} F^{\beta} K^{\gamma} z^{\delta}\right) \neq 0$ for some $0 \leq \alpha, \beta<t$. Suppose that $\alpha>\beta$, then $\beta+1 \leq \alpha<t$. By (4.4), we have

$$
\begin{aligned}
0 & =\lambda\left(E^{\alpha} F^{\beta+1} K^{\beta} z^{\beta}\right)=\sum_{\substack{0 \leq k \leq \alpha \\
0 \leq \ell \leq \bar{\beta}+1}} A_{\alpha, \beta+1, k, \ell} \lambda\left(E^{\alpha-k} F^{\beta+1-\ell} K^{\beta+k} z^{\beta}\right) E^{k} F^{\ell} K^{\ell-1} z^{\ell-1} \\
& =A_{\alpha, \beta+1,0,1} \lambda\left(E^{\alpha} F^{\beta} K^{\beta} z^{\beta}\right) F \neq 0,
\end{aligned}
$$

which is a contradiction! Similarly, that $\alpha$ is not less than $\beta$ is also impossible! This proves that if $\lambda\left(E^{\alpha} F^{\beta} K^{\gamma} z^{\delta}\right) \neq 0$, then $\alpha=\beta=\gamma=\delta$.

Let $\lambda\left(E^{\alpha} F^{\alpha} K^{\alpha} z^{\alpha}\right) \neq 0$ for some $0 \leq \alpha<t$. If $\alpha<t-1$, then we would derive a contradiction by considering $\lambda\left(E^{\alpha+1} F^{\alpha+1} K^{\alpha-1} z^{\alpha}\right)$. Therefore, $\alpha$ has to be $t-1$. In other words, $\lambda$ is a scalar multiple of $\overline{E^{t-1} F^{t-1} K^{t-1} z^{t-1}}$.

(4) The proof is similar to the proof of (3).

(5) Let $\lambda$ be a non-zero right integral for $s \ell_{q, z}^{t}(2)^{*}$. By (2.5), we have

$$
\lambda(h) g=\sum_{(h)} \lambda\left(h_{(2)}\right) h_{(1)} \quad \text { for } h \in s \ell_{q, z}^{t}(2)^{*}
$$


Letting $\lambda=\overline{E^{t-1} F^{t-1} K^{t-1} z^{t-1}}$ and $h=E^{t-1} F^{t-1} K^{t-1} z^{t-1}$, we get

$$
\begin{aligned}
& g=\sum_{\substack{0 \leq k \leq t-1 \\
0 \leq \ell \leq t-1}} q^{k(t-1-k)+(t-1-\ell)(\ell-2 k)} \\
& \quad \times\left(\begin{array}{c}
t-1 \\
k
\end{array}\right)\left(\begin{array}{c}
t-1 \\
\ell
\end{array}\right) E^{t-1-k} F^{t-1-\ell} K^{k+t-1} z^{t-1} \lambda\left(E^{k} F^{\ell} K^{\ell} z^{\ell}\right) \\
& =K^{t-2} z^{t-1} .
\end{aligned}
$$

5. The Determination of The TRACE SPACE FOR $s \ell_{q, z}^{t}(2)$

In this section, we determine the trace space for $s \ell_{q, z}^{t}(2)$ with an odd $t$. Because of the relation between the $m$ th trace space and the trace space given in Proposition 3.1 , it suffices to determine $\mathcal{T}_{1}\left(s \ell_{q, z}^{t}(2)^{*}\right)$, which consists of all functionals $p$ with the properties

$$
\begin{gathered}
p(x y)=p\left(S^{2}(y) x\right) \quad \text { for } x, y \in s \ell_{q, z}^{t}(2), \\
p\left(G^{2} x\right)=p(S(x)) \quad \text { for } x \in s \ell_{q, z}^{t}(2),
\end{gathered}
$$

where $G:=K^{-1} z^{-1-\xi}$ and $\xi \in \mathbb{Z} / t \mathbb{Z}$.

Let $x=E^{m} F^{n} K^{a} z^{b}$, where $0 \leq m, n, a, b<t$. Then

$$
x G=E^{m} F^{n} K^{a-1} z^{b-1-\xi}, \quad G x=q^{2(n-m)} E^{m} F^{n} K^{a-1} z^{b-1-\xi} .
$$

Since $p(x G)=p(G x)$ by $(5.1)$, we get $\left(q^{2(n-m)}-1\right) p\left(E^{m} F^{n} K^{a-1} z^{b-1-\xi}\right)=0$, which implies that

$$
p\left(E^{m} F^{n} K^{a} z^{b}\right) \neq 0 \Longrightarrow m=n, \quad \text { where } 0 \leq m, n, a, b<t .
$$

Let $x=E^{n} F^{n} K^{a} z^{b}$. Then, by Lemma 4.1, we get

$$
S(x)=\sum_{0 \leq j \leq i \leq n}(-1)^{i}\left(\begin{array}{c}
n \\
i
\end{array}\right)^{2}[i] ! \frac{q^{\frac{(2 j-i)(i-1)}{2}}}{\left(q-q^{-1}\right)^{i}}\left(\begin{array}{c}
i \\
j
\end{array}\right) E^{n-i} F^{n-i} K^{2 j-i-a} z^{j-i+n-b}
$$

It is clear that $x G^{2}=E^{n} F^{n} K^{a-2} z^{b-2-2 \xi}$. Hence, by (5.2), we have

$$
\begin{aligned}
& p\left(E^{n} F^{n} K^{a-2} z^{b-2-2 \xi}\right) \\
= & \sum_{0 \leq j \leq i \leq n}(-1)^{i}\left(\begin{array}{c}
n \\
i
\end{array}\right)^{2}[i] ! \frac{q^{\frac{(2 j-i)(i-1)}{2}}}{\left(q-q^{-1}\right)^{i}}\left(\begin{array}{l}
i \\
j
\end{array}\right) p\left(E^{n-i} F^{n-i} K^{2 j-i-a} z^{j-i+n-b}\right),
\end{aligned}
$$

where $n, a, b \in \mathbb{Z} / t \mathbb{Z}$. 
Letting $x=E^{m} F^{n} K^{a} z^{b}$ and $y=E^{m^{\prime}} F^{n^{\prime}} K^{a^{\prime}} z^{b^{\prime}}$ in (5.1) and using Lemma 4.1, we have

$$
\begin{aligned}
& q^{2 a\left(m^{\prime}-n^{\prime}\right)} \sum_{0 \leq i \leq \min \left(n, m^{\prime}\right)}(-1)^{i}\left(\begin{array}{c}
n \\
i
\end{array}\right)\left(\begin{array}{c}
m^{\prime} \\
i
\end{array}\right)[i] !^{2}\left(\begin{array}{ccc}
K, & z ; & n-m^{\prime}-1+i \\
i &
\end{array}\right) \\
& \times p\left(E^{m+m^{\prime}-i} F^{n+n^{\prime}-i} K^{a+a^{\prime}} z^{b+b^{\prime}}\right) \\
& =q^{2\left(n^{\prime}-m^{\prime}\right)+2 a^{\prime}(m-n)} \sum_{0 \leq i \leq \min \left(n^{\prime}, m\right)}(-1)^{i}\left(\begin{array}{c}
n^{\prime} \\
i
\end{array}\right)\left(\begin{array}{c}
m \\
i
\end{array}\right)[i] !^{2}\left(\begin{array}{ccc}
K, & z ; & n^{\prime}-m-1+i \\
i &
\end{array}\right) \\
& \times p\left(E^{m+m^{\prime}-i} F^{n+n^{\prime}-i} K^{a+a^{\prime}} z^{b+b^{\prime}}\right),
\end{aligned}
$$

where $m, n, a, b, m^{\prime}, n^{\prime}, a^{\prime}, b^{\prime} \in \mathbb{Z} / t \mathbb{Z}$. Note that $n$ (or $\left.m^{\prime}\right)$ in the notation $\min \left(n, m^{\prime}\right)$ is regarded as the least residue of $n$ (or $m^{\prime}$ ) modulo $t$.

Now we choose the minimal $m_{0} \in\{0,1, \ldots, t-1\}$ such that $p\left(E^{m_{0}} F^{m_{0}} K^{\alpha} z^{\beta}\right) \neq 0$ for some $\alpha, \beta \in \mathbb{Z} / t \mathbb{Z}$. Note that $\alpha$ and $\beta$ may be not unique. In other words,

$$
p\left(E^{m} F^{m} K^{a} z^{b}\right)=0 \quad \text { if } 0 \leq m<m_{0} \text { and } a, b \in \mathbb{Z} / t \mathbb{Z}
$$

Furthermore, we choose $m, n, a, b, m^{\prime}, n^{\prime}, a^{\prime}, b^{\prime} \in \mathbb{Z} / t \mathbb{Z}$ such that $m=1, n=0$, $m+m^{\prime}=m_{0}=n+n^{\prime}, a+a^{\prime}=\alpha$ and $b+b^{\prime}=\beta$. It follows from (5.5) and (5.6) that $\alpha \equiv-1 \bmod t$. This proves that

$$
p\left(E^{m_{0}} F^{m_{0}} K^{\alpha} z^{\beta}\right)=0 \quad \text { if } \alpha \not \equiv-1 \quad \bmod t .
$$

Suppose that $m_{0}<t-1$. Then $m_{0}+1 \leq t-1$. Letting $m=1, n=0, m^{\prime}=m_{0}$ and $n^{\prime}=m_{0}+1$ in (5.5), we get

$$
\begin{aligned}
q^{-2 a} p\left(E^{m_{0}+1} F^{m_{0}+1} K^{a+a^{\prime}} z^{b+b^{\prime}}\right) & \\
= & q^{2+2 a^{\prime}} p\left(E^{m_{0}+1} F^{m_{0}+1} K^{a+a^{\prime}} z^{b+b^{\prime}}\right) \\
& -\frac{q^{2+2 a^{\prime}+m_{0}}\left[m_{0}+1\right]}{q-q^{-1}} p\left(E^{m_{0}} F^{m_{0}} K^{a+a^{\prime}+1} z^{b+b^{\prime}}\right) \\
& +\frac{q^{2+2 a^{\prime}+m_{0}}\left[m_{0}+1\right]}{q-q^{-1}} p\left(E^{m_{0}} F^{m_{0}} K^{a+a^{\prime}-1} z^{b+b^{\prime}-1}\right)
\end{aligned}
$$

where $a, b, a^{\prime}, b^{\prime} \in \mathbb{Z} / t \mathbb{Z}$.

Taking $a+a^{\prime}$ to be $t-2$ in (5.8), we get

$$
p\left(E^{m_{0}+1} F^{m_{0}+1} K^{t-2} z^{b}\right)=-\frac{q^{m_{0}-1}\left[m_{0}+1\right]}{\left(q-q^{-1}\right)^{2}} p\left(E^{m_{0}} F^{m_{0}} K^{t-1} z^{b}\right) \quad \text { for } b \in \mathbb{Z} / t \mathbb{Z} \text {. }
$$

Taking $a+a^{\prime}$ to be 0 in (5.8), we get

$$
p\left(E^{m_{0}+1} F^{m_{0}+1} z^{b}\right)=-\frac{q^{1-m_{0}}\left[m_{0}+1\right]}{\left(q-q^{-1}\right)^{2}} p\left(E^{m_{0}} F^{m_{0}} K^{t-1} z^{b-1}\right) \quad \text { for } b \in \mathbb{Z} / t \mathbb{Z} .
$$

Letting $n=m_{0}+1$ and $a=0$ in (5.4), we get

$$
\begin{array}{r}
p\left(E^{m_{0}+1} F^{m_{0}+1} K^{t-2} z^{b-2-2 \xi}\right)=p\left(E^{m_{0}+1} F^{m_{0}+1} z^{1+m_{0}-b}\right) \\
-\frac{\left[m_{0}+1\right]^{2}}{q-q^{-1}} p\left(E^{m_{0}} F^{m_{0}} K^{t-1} z^{m_{0}-b}\right) \quad \text { for } b \in \mathbb{Z} / t \mathbb{Z} .
\end{array}
$$


Using (5.9) and (5.10), it follows from (5.11) that

$$
p\left(E^{m_{0}} F^{m_{0}} K^{t-1} z^{b-2-2 \xi}\right)=\left(q^{2-2 m_{0}}+q^{2}-q^{-2 m_{0}}\right) p\left(E^{m_{0}} F^{m_{0}} K^{t-1} z^{m_{0}-b}\right),
$$

where $b \in \mathbb{Z} / t \mathbb{Z}$. Replacing $b-2-2 \xi$ by $b$ in the equation above gives

$$
p\left(E^{m_{0}} F^{m_{0}} K^{t-1} z^{b}\right)=\left(q^{2-2 m_{0}}+q^{2}-q^{-2 m_{0}}\right) p\left(E^{m_{0}} F^{m_{0}} K^{t-1} z^{m_{0}-b-2 \xi-2}\right),
$$

where $b \in \mathbb{Z} / t \mathbb{Z}$. This implies that

$$
\left(q^{2-2 m_{0}}+q^{2}-q^{-2 m_{0}}\right)^{2}=1,
$$

which is impossible for odd $t$. Therefore, $m_{0}=t-1$. Combining this fact with (5.3) and (5.7), we have proved that

$$
p\left(E^{m} F^{n} K^{a} z^{b}\right) \neq 0 \Longrightarrow m \equiv n \equiv a \equiv t-1 \quad \bmod t .
$$

Letting $n=t-1$ and $a=1$ in (5.4), we get

$$
p\left(E^{t-1} F^{t-1} K^{t-1} z^{\beta}\right)=p\left(E^{t-1} F^{t-1} K^{t-1} z^{-\beta-2 \xi-3}\right) \quad \text { for } \beta \in \mathbb{Z} / t \mathbb{Z} .
$$

Hence, for some $a_{\beta} \in \mathbb{C}$,

$$
\begin{aligned}
p & =\sum_{\beta \in \mathbb{Z} / t \mathbb{Z}} a_{\beta}\left(\overline{E^{t-1} F^{t-1} K^{t-1} z^{\beta}}+\overline{E^{t-1} F^{t-1} K^{t-1} z^{-\beta-2 \xi-3}}\right) \\
& =\sum_{j \in \mathbb{Z} / t \mathbb{Z}} a_{j}\left(\overline{E^{t-1} F^{t-1} K^{t-1} z^{j-1}}+\overline{E^{t-1} F^{t-1} K^{t-1} z^{-j-2 \xi-2}}\right) \\
& =\sum_{j \in \mathbb{Z} / t \mathbb{Z}} a_{j}\left\{\lambda \leftarrow\left(z^{-j}+z^{j+2 \xi+1}\right)\right\},
\end{aligned}
$$

where $\lambda=\overline{E^{t-1} F^{t-1} K^{t-1} z^{t-1}}$ is a non-zero right integral for $s \ell_{q, z}^{t}(2)^{*}$ and we have used the following fact:

$$
\overline{E^{t-1} F^{t-1} K^{t-1} z^{t-1}} \leftarrow z^{u}=\overline{E^{t-1} F^{t-1} K^{t-1} z^{t-1-u}} \quad \text { for } u \in \mathbb{Z} / t \mathbb{Z} .
$$

This proves that every $p \in \mathcal{T}_{1}\left(s \ell_{q, z}^{t}(2)^{*}\right)$ is a $\mathbb{C}$-linear combination of the elements in the set

$$
\left\{\lambda<\left(z^{-j}+z^{j+2 \xi+1}\right) \mid \quad j \in \mathbb{Z} / t \mathbb{Z}\right\} .
$$

One of the maximal linearly independent subsets of the set above is given by

$$
\left\{\lambda<z^{-\frac{t-2 \xi-1}{2}}, \quad \lambda<\left(z^{-m_{i}}+z^{\left.m_{i}+2 \xi+1\right)}\right) \mid \quad 1 \leq i \leq \frac{t-1}{2}\right\},
$$

where $\left\{-\frac{t-2 \xi-1}{2},-m_{1}, m_{1}+2 \xi+1, \ldots,-m_{\frac{t-1}{2}}, m_{\frac{t-1}{2}}+2 \xi+1\right\}$ is a complete residue system modulo $t$. Hence, the set given by $(5.12)$ is a basis of $\mathcal{T}_{1}\left(s \ell_{q, z}^{t}(2)^{*}\right)$. By Corollary $3.7, \Omega(\langle z\rangle)=\frac{t+1}{2}$. So

$$
\operatorname{dim}\left(\mathcal{T}_{1}\left(s \ell_{q, z}^{t}(2)^{*}\right)\right)=\frac{t-1}{2}+1=\frac{t+1}{2}=\Omega(<z>),
$$

which is the lower bound given in Proposition 3.6. This completes the proof of Proposition 3.6.

We finish this paper by summarizing the result about the trace space for $s \ell_{q, z}^{t}(2)$ as follows: 
Proposition 5.1. Let $\left(s \ell_{q, z}^{t}(2), S, \mathcal{R}_{\left(d_{\alpha \beta}\right)_{0 \leq \alpha, \beta<t}}, K^{-1} z^{-1-\xi}\right)$ be the quantum Hopf algebra in Proposition 4.2. Let $\lambda$ be a non-zero right integral for $s \ell_{q, z}^{t}(2)^{*}$. If $t$ is odd, then the dimension of the trace space $\mathcal{T}_{0}\left(s \ell_{q, z}^{t}(2)^{*}\right)$ is $\frac{t+1}{2}$ and the set

$$
\left\{\lambda<K^{-1} z^{-\frac{t+1}{2}}, \quad \lambda<K^{-1}\left(z^{-m_{i}-\xi-1}+z^{\left.m_{i}+\xi\right)}\right) \mid \quad 1 \leq i \leq \frac{t-1}{2}\right\}
$$

is a $\mathbb{C}$-basis of the trace space, where

$$
\left\{-\frac{t-2 \xi-1}{2},-m_{1}, m_{1}+2 \xi+1, \ldots,-m_{\frac{t-1}{2}}, m_{\frac{t-1}{2}}+2 \xi+1\right\}
$$

is a complete residue system modulo $t$.

\section{ACKNOWLEDGMENT}

I would like to thank Professor J. B. Carrell for his support and Professor R. V. Moody and Professor D. Rolfsen for useful conversations. Also, I would like to thank the referee for his or her comments, which have helped the author to clarify some mathematical points in the paper.

\section{REFERENCES}

1. V. G. Drinfeld, On almost cocommutative Hopf algebras, Leningrad Math. J. 1, No.2 (1990), 321-342.

2. L. H. Kauffman, Gauss codes, quantum groups and ribbon Hopf algebras, Reviews in Math. Phys. 5, no.4 (1993), 735-773. MR 94k:57013

3. L. H. Kauffman, Hopf algebras and invariants of 3-manifolds, Jour. of Pure and Applied Algebra 100 (1995), 73-92. MR 96h:57014

4. R. Kirby and P. Melvin, The 3-manifold invariants of Witten and Reshetikhin-Turaev for $\operatorname{sll}(2, \mathbb{C})$, Inv. Math. 105 (1991), 473-545. MR 92e:57011

5. R. G. Larson and M. E. Sweedler, An associative orthogonal bilinear form for Hopf algebras, Amer. J. Math. 91 (1969), 75-94. MR 39:1523

6. K. Liu, A family of new universal $R$-matrices, to appear.

7. D. E. Radford, On Kauffman's knot invariants arising from finite-dimensional Hopf algebras, Lecture Notes in Pure and Applied Math. 158 (1992), 205-266. MR 96g:57013

8. D. E. Radford, The trace function and Hopf algebras, J. of Algebra. 163 (1994), 583-622. MR 95e: 16039

9. D. E. Radford, The order of the antipode of a finite-dimensional Hopf algebra is finite, Amer. J. of Math. 98 (1976), 333-355. MR 53:10852

10. N. Reshetikhin and V.G.Turaev, Invariants of 3-manifolds via link polynomials and quantum groups, Inv. Math. 103 (1991), 547-597. MR 92b:57024

11. M. E. Sweedler, Hopf algebra, Math. Lecture Notes Series, Benjamin, New York, 1969. MR 40:5705

Department of Mathematics, The University of British Columbia, Vancouver, BC, CANADA V6T 1 Z2 